\title{
Cavity QED implementation of the discrete quantum Fourier transform
}

\author{
Marlan O. Scully \\ Department of Physics and Institute for Quantum Studies, Texas A\&M University, College Station, Texas 77843-4242 \\ and Max-Planck-Institut für Quantenoptik, D-85748 Garching, Germany \\ M. Suhail Zubairy \\ Department of Physics and Institute for Quantum Studies, Texas A\&M University, College Station, Texas 77843-4242 \\ and Department of Electronics, Quaid-i-Azam University, Islamabad, Pakistan
}

(Received 24 November 2001; published 8 May 2002)

\begin{abstract}
We present a scheme for the implementation of the discrete quantum Fourier transform using cavity quantum electrodynamics. In the proposed scheme a series of atoms whose atomic coherence carries the input state passes through a series of cavities and classical field, and the resulting state in the cavities is the quantum Fourier transform of the input state.
\end{abstract}

DOI: 10.1103/PhysRevA.65.052324

PACS number(s): 03.67.Lx

A dramatic example of the potential applications of a quantum computer is the factorization of a composite number via Shor's algorithm, and the quantum Fourier transform (QFT) lies at the heart of the algorithm [1]. In this paper we present a scheme based on cavity quantum electrodynamics to implement the QFT. The complexity of implementing the discrete Fourier transform on a quantum computer arises due to the large number of quantum logic gates interacting between several qubits.

Recently a number of approaches have been proposed for the implementation of quantum logic gates and simple quantum algorithms. One approach is based on nuclear-magneticresonance techniques where logic gates and search algorithms have been experimentally demonstrated at room temperature [2]. However, questions have been raised concerning the appearance of entanglement in the physical state at any stage of the experiment [3]. Another promising approach is based on cavity quantum electrodynamics (QED) [4] where quantum logic gates have been experimentally realized $[5,6]$ and proposals have been made to implement search algorithms [7] based on these considerations. Such schemes are relatively insensitive to thermal decoherence effects.

In this paper we propose an implementation of the QFT via a cavity QED scheme. The basic building blocks are the one-bit unitary gate and two-bit quantum phase gate. The proposed scheme relies on the passage of a series of suitably chosen atoms through a sequence of classical fields and high$Q$ cavities. by

The QFT maps each state $|a\rangle$ into a superposition given

$$
N_{\mathrm{QFT}}|a\rangle=\frac{1}{\sqrt{2^{q}}} \sum_{c=0}^{2^{q}-1} e^{2 \pi i a c / 2^{q}}|c\rangle,
$$

where $q$ is the number of qubits. Our aim is the implementation of this mapping using quantum logic gates. As quantum logic gates operate on qubits, we consider a representation of state $|a\rangle$ in terms of qubits. Thus, for example, $|9\rangle$ $\equiv|1,0,0,1\rangle$. For $2^{q}>a>2^{q-1}$, we therefore require $q$ qubits to represent $|a\rangle$.
A universal quantum computer can be built from only two gates, namely, a one-bit unitary gate and a two-bit conditional quantum phase gate. The one-bit quantum gate for the $i$ th qubit is given by

$$
U_{\theta, \phi}^{i}=\left(\begin{array}{cc}
\cos \theta & -i e^{-i \phi} \sin \theta \\
-i e^{i \phi} \sin \theta & \cos \theta
\end{array}\right),
$$

and a convenient representation of $U_{\theta, \phi}$ in terms of Pauli spin matrices is given by

$$
U_{\theta, \phi}=\cos (\theta) 1-i \cos (\phi) \sin (\theta) \sigma_{x}-i \sin (\phi) \sin (\theta) \sigma_{y} .
$$

The transformation for a two-bit quantum phase gate for the $j$ th and $k$ th qubits is given by $Q_{\eta}^{j k}\left|\alpha_{j}, \beta_{k}\right\rangle$ $=\exp \left(i \eta \delta_{\alpha_{j}, 1} \delta_{\beta_{k}, 1}\right)\left|\alpha_{j}, \beta_{k}\right\rangle$, where $\left|\alpha_{j}\right\rangle$ and $\left|\beta_{k}\right\rangle$ stand for the basis states $|0\rangle$ or $|1\rangle$ of the qubits. Thus the quantum phase gate introduces a phase $\eta$ only when both the qubits in the input states are 1 . A representation of the quantum phase gate is given by the operator

$$
\begin{aligned}
Q_{\eta}^{j k}= & \left|0_{j}, 0_{k}\right\rangle\left\langle 0_{j}, 0_{k}|+| 0_{j}, 1_{k}\right\rangle\left\langle<0_{j}, 1_{k}\right| \\
& +\left|1_{j}, 0_{k}\right\rangle\left\langle 1_{j}, 0_{k}\left|+e^{i \eta}\right| 1_{j}, 1_{k}\right\rangle\left\langle 1_{j}, 1_{k}\right|,
\end{aligned}
$$

and since $|0\rangle\langle 0|=\left(1+\sigma_{z}\right) / 2$ and $|1\rangle\langle 1|=\left(1-\sigma_{z}\right) / 2$, Eq. (4) has the matrix representation

$$
Q_{\eta}=1_{1} 1_{2}-\frac{1}{4}\left(1-e^{i \eta}\right)\left(1_{1} 1_{2}-1_{1} \sigma_{z 2}-\sigma_{z 1} 1_{2}+\sigma_{z 1} \sigma_{z 2}\right) .
$$

The discrete Fourier transform (1) is depicted in terms of the above one-bit unitary gate and two-bit phase gate in Fig. 1(a). Thus, for example, the transformation for $q=3$ in Eq. (1) is generated by

$$
A_{0} B_{01} B_{02} A_{1} B_{12} A_{2},
$$

where $A_{i} \equiv U_{\pi / 4, \pi / 2}^{i}$ and $B_{j k} \equiv Q_{\pi / 2^{k-j}}^{j k}$. The QFT for $q$ qubits requires $q$ one-bit gates and $q(q-1) / 2$ two-bit phase gates. 


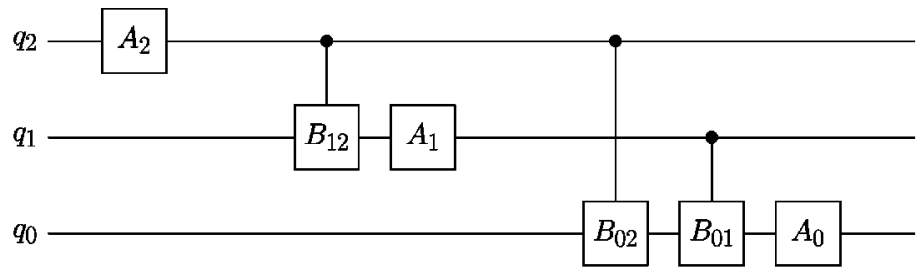

(0) (1) (2)

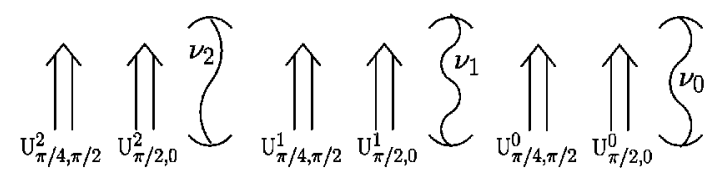

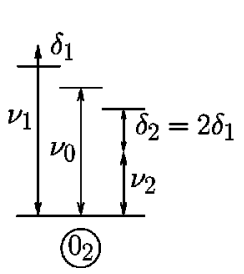
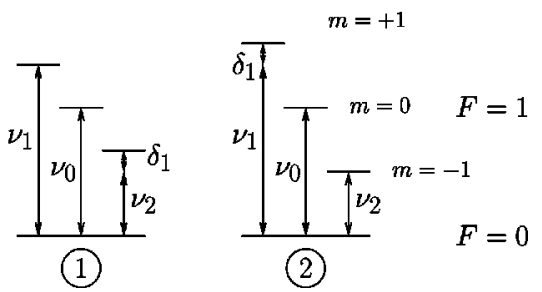

FIG. 1. (a) Circuit diagram for the QFT with three qubits. Here $A_{i} \equiv U_{\pi / 4, \pi / 2}^{i}$ is the one-bit gate and $B_{j k} \equiv Q_{\pi / 2^{k-j}}^{j k}$ is the two-bit quantum phase gate. (b) The physical implementation of the QFT. Here three atoms labeled 0, 1, and 2 interact with a sequence of classical fields and cavities. (c) The atomic level scheme for the three atoms as obtained under the influence of magnetic fields. For the atom labeled 0, the level spacings are chosen different in the two cavities resonant with frequencies $\nu_{1}$ and $\nu_{2}$.
This indicates the complexity involved in the implementation of discrete Fourier transform. We also note that the initial state $|a\rangle$ may in general be entangled. Before describing an implementation of the QFT, we first describe our quantum optical implementation of one-bit unitary gate and a two-bit phase gate.

The unitary one-bit gate can be simply implemented by passing a two-level atom through a classical field. The Hamiltonian, in the rotating-wave approximation and the interaction picture, is given by

$$
\mathcal{V}=\frac{\hbar \Omega}{2}\left(e^{-i \phi}|a\rangle\left\langle b\left|+e^{i \phi}\right| b\right\rangle\langle a|\right),
$$

where $\Omega$ is the Rabi frequency and we assume $|a\rangle$ to be the excited state and $|b\rangle$ to be the ground state. The corresponding time-evolution unitary operator is given by

$$
\begin{aligned}
U_{I}(t)= & \exp \left(-\frac{i}{\hbar} \mathcal{V} t\right) \\
= & \cos (\Omega t / 2)(|a\rangle\langle a|+| b\rangle\langle b|) \\
& -i \sin (\Omega t / 2)\left(e^{-i \phi}|a\rangle\left\langle b\left|+e^{i \phi}\right| b\right\rangle\langle a|\right) .
\end{aligned}
$$

This corresponds to the one-bit unitary gate (2) with $\theta$ $=\Omega t / 2$.

The quantum phase gate can be implemented via dispersive coupling of a three-level atom with a cavity field [5]. We assume a three-level atom in $V$ configuration with $|b\rangle$ to be the ground state and $|a\rangle$ and $|c\rangle$ to be the excited states. The atomic transition $|a\rangle \rightarrow|b\rangle$ is completely off resonant with the cavity field whereas the transition $|c\rangle \rightarrow|b\rangle$ is somewhat off resonant with the cavity field (i.e., is dispersively coupled to the cavity). The effective Hamiltonian for the atom and the cavity field is given by [8]

$$
\mathcal{H}_{e f f}=-\frac{\hbar g^{2}}{\Delta}\left(a a^{\dagger}|c\rangle\left\langle c\left|-a^{\dagger} a\right| b\right\rangle\langle b|\right),
$$

where $g$ is a coupling coefficient, $a$ and $a^{\dagger}$ are destruction and creation operators for the field state inside the cavity, and $\Delta=\omega_{c b}-\nu$ is the detuning. For an initial state in which the atom-field state is

$$
\frac{1}{2}(|0, a\rangle+|0, b\rangle+|1, a\rangle+|1, b\rangle),
$$

the resulting entangled state after the passage of the atom through the cavity is

$$
\frac{1}{2}\left(|0, a\rangle+|0, b\rangle+|1, a\rangle+e^{i \eta}|1, b\rangle\right),
$$

where $\eta=g^{2} \tau / \Delta$. The net result is that there is a phase change only when there is one photon inside the cavity and the atom is in the state $|b\rangle$. The interaction time $\tau$ and the detuning $\Delta$ can be appropriately chosen for a given choice of $\eta$. For atomic states such that $|a\rangle$ and $|b\rangle$ correspond to qubits $|0\rangle$ and $|1\rangle$, respectively, this represents the quantum phase gate discussed above and experimentally implemented in Ref. [5].

Next we consider the implementation of the QFT using these basic building blocks. We shall consider only the case of $q=3$. A generalization to higher values of $q$ can be carried out along similar lines.

The initial state is characterized by the internal states of the atoms labeled 0,1 , and 2 [Fig. 1(b)]. The atoms consist of a ground state $|b\rangle$ and three excited state $a_{1}, a_{0}$, and $a_{2}$. The excited states could be the magnetic sublevels whose spacings can be changed by applying appropriate magnetic fields. For all the atoms, the ground state $|b\rangle$ corresponds to the qubit $|1\rangle$ and $\left|a_{i}\right\rangle$ to be the qubit $|0\rangle$ for the $i$ th atom with 
$i=0,1,2$. Thus, for example, an initial state $|101\rangle$ would imply an initial state $\left|b, a_{1}, b\right\rangle$ for the atoms. The initial state can in general be an entangled state as would be required, for example, in the implementation of Shor's algorithm. Here we do not address the important question concerning how such an entangled state can be prepared. We focus instead on the implementation of the QFT of a given initial state.

Having set the stage, we proceed to describe the present QFT implementation. The atoms pass through a sequence of three classical fields that implement the one-bit unitary gates

$$
A_{i} \equiv U_{\pi / 4, \pi / 2}^{i}
$$

and a sequence of three cavities labeled $C_{2}, C_{1}$, and $C_{0}$ resonant with frequencies $\nu_{2}, \nu_{1}$, and $\nu_{0}$, respectively, which serve to implement quantum phase gates $B_{j k}$. We also need another sequence of classical fields $U_{\pi / 2,0}^{i}$ in order to flip the states $\left|a_{i}\right\rangle$ and $|b\rangle$ before $i$ th atom enters the cavity $C_{i}$. This transformation is required because we label the ground state $|b\rangle$ as $|1\rangle$ and the excited states $\left|a_{i}\right\rangle$ as $|0\rangle$ and a switching of atomic states before entering the cavity would ensure that the qubits $|1\rangle$ and $|0\rangle$ lead to photon states $|1\rangle$ and $|0\rangle$, respectively, inside the cavity. The magnetic fields are applied in such a way that, for the $i$ th atom, the $\left|a_{i}\right\rangle$ $\rightarrow|b\rangle$ transition is resonant with the corresponding classical field $U_{\theta, \phi}^{i}$ and is resonant with the cavity field in $C_{i}$. In addition, for the zeroth atom, the magnetic field in cavities 1 and 2 are such that the detuning with respect to the cavity fields are $\delta_{1}$ for $\left|a_{1}\right\rangle \rightarrow|b\rangle$ transition in $C_{1}$ and $\delta_{2}=2 \delta_{1}$ for $\left|a_{2}\right\rangle \rightarrow|b\rangle$ transition in $C_{2}$, respectively [see Fig. 1(c)].

Thus when the $j$ th atom is passing through the $k$ th cavity with $j \neq k$, a quantum phase gate is implemented with $\eta$ $=g^{2} \tau / \delta_{k-j}$ where $g$ is the coupling constant and $\tau$ is the interaction time. We have taken $g$ and $\tau$ to be equal for all atoms and for all levels for the sake of simplicity. The interaction times, the coupling constants and the detunings $\delta_{i}$ are chosen such that $g^{2} \tau / \delta_{k-j}=\pi / 2^{k-j}$. The interaction times and coupling constants are also chosen in such a way that if the $j$ th atom is passing through the $j$ th cavity, it transfers its coherence to the cavity and leaves in the ground state $\left|b_{j}\right\rangle$.

With these conditions it is easy to see how the QFT logic can be implemented. Initially all the cavities are prepared in the vacuum state, i.e., $|0,0,0\rangle$. The first atom (denoted as 2 because it serves to encode the coefficient of $2^{2}$ ) passes through a classical field that is resonant with the $\left|a_{2}\right\rangle \rightarrow|b\rangle$ transition. The Rabi frequency and the interaction time are chosen such that the one-bit gate $A_{2} \equiv U_{\pi / 4, \pi / 2}^{2}$ is implemented. Atom 2 then passes through the classical field corresponding to $U_{\pi / 2,0}^{2}$ in order to flip the states $\left|a_{2}\right\rangle$ and $|b\rangle$.
Finally, atom 2 passes through the cavity $C_{2}$ that is also resonant with the $\left|a_{2}\right\rangle \rightarrow|b\rangle$ transition and the interaction time is chosen such that the atom transfers its coherence to the cavity, exits the cavity in the ground state $\left|b_{2}\right\rangle$, and is discarded.

For the second atom, labeled as "1," the applied magnetic field leads to the level spacings such that $\left|a_{1}\right\rangle \rightarrow|b\rangle$ is tuned to $\nu_{1}$ and $\left|a_{2}\right\rangle \rightarrow|b\rangle$ transition is detuned with respect to $\nu_{2}$ by an amount $\delta_{1}$. Atom 1 first passes through the cavity $C_{2}$, and makes a quantum phase gate as discussed above. This is followed by a passage through a classical field resonant with $\left|a_{1}\right\rangle \rightarrow|b\rangle$ transition and implement the one-bit gate $A_{2}$ $\equiv U_{\pi / 4, \pi / 2}^{1}$. The atom 1 then passes through a classical field to flip the states $\left|a_{1}\right\rangle$ and $|b\rangle$. Finally, it passes through the cavity $C_{1}$ that is resonant with the $\left|a_{1}\right\rangle \rightarrow|b\rangle$ transition and transfers its coherence to the cavity and leaves in the ground state $|b\rangle$.

For the third atom, labeled as " 0 ," the applied magnetic fields are different in the two cavities $C_{2}$ and $C_{1}$ and are such that, during the passage of the atom 0 through these cavities, phase gates with appropriate phase shifts are implemented [Fig. 1(c)]. Thus the atom 0, after implementing phase gates via interaction with the fields in cavities $C_{2}$ and $C_{1}$, forms a one-bit gate with a classical field, flips the states $\left|a_{0}\right\rangle$ and $|b\rangle$, and leaves the resulting atomic coherence in the cavity $C_{0}$. The quantum state of the fields in the three cavities is the resulting quantum Fourier transform of the initial states of the three atoms.

The readout would require three more atoms that are resonant with the cavity fields. These atoms are sent in the respective cavities in the ground state such that, after the passage through the cavity, a ground state would correspond to the output zero and the excited state would correspond to the output 1 .

The proposed scheme can be extended to an arbitrary number $n$ of qubits. However, that would require $n$ cavities and a configuration of atoms in which a single level is coupled to atoms with $n$ levels whose level spacings can be appropriately manipulated with external fields. A simpler scheme with a single cavity can be designed in which atoms interact with various modes of the cavity. The level spacings are modulated by the field inside the cavity and are such that, at a given time, the atom interacts with only one mode, either resonantly or dispersively as the situation requires.

The authors gratefully acknowledge the support from the Air Force Research Laboratory (Rome, New York), DARPAQuIST, TAMU Telecommunication and Informatics Task Force (TITF) Initiative, the Office of Naval Research, and the Welch Foundation.
[1] P. W. Shor, in Proceedings of the 35th Annual Symposium on Foundations of Computer Science, Santa Fe, NM, 1994, edited by S. Goldwasser (IEEE Computer Society Press, Los Alamitos, CA, 1994), p. 124; SIAM J. Comput. 26, 1484 (1997).

[2] I. L. Chuang, N. Gershenfeld, and M. Kubinec, Phys. Rev. Lett. 80, 3408 (1998); J. A. Jones, M. Mosca, and R. H.
Hansen, Nature (London) 393, 344 (1998); J. A. Jones, Science 280, 229 (1998).

[3] S. L. Braunstein, C. M. Caves, R. Jozsa, N. Linden, S. Popescu, and R. Schack, Phys. Rev. Lett. 83, 1054 (1999).

[4] D. Meschede, H. Walther, and G. Muller, Phys. Rev. Lett. 54, 551 (1985); S. Haroche and J. M. Raimond, in Advances in 
Atomic, Molecular, and Optical Physics, edited by D. R. Bates and B. Bederson (Academic, New York, 1985), Vol. 20, p. 350; K. An, J. J. Childs, R. R. Dasari, and M. S. Feld, Phys. Rev. Lett. 73, 3375 (1994); G. Raithel, C. Wagner, H. Walther, L. M. Narducci, and M. O. Scully, in Advances in Atomic, Molecular, and Optical Physics, edited by P. Berman (Academic, New York, 1994), Suppl. 2, p. 57; C. J. Hood, M. S. Chapman, T. W. Lynn, and H. J. Kimble, Phys. Rev. Lett. 80, 4157 (1998).

[5] A. Rauschenbeutel, G. Nogues, S. Osnaghi, P. Bertet, M.
Brune, J. M. Raimond, and S. Haroche, Phys. Rev. Lett. 83, 5166 (1999).

[6] Q. A. Turchette, C. J. Hood, W. Lange, H. Mabuchi, and H. J. Kimble, Phys. Rev. Lett. 75, 4710 (1995).

[7] M. O. Scully and M. S. Zubairy, Proc. Natl. Acad. Sci. U.S.A. 98, 9490 (2001).

[8] M. Brune, S. Haroche, V. Lefevre, J. M. Raimond, and N. Zagury, Phys. Rev. Lett. 65, 976 (1990); see also, M. O. Scully and M. S. Zubairy, Quantum Optics (Cambridge University Press, Cambridge, England, 1997), Chap. 19. 